\title{
Plant surface wax affects parasitoid's response to host footprints
}

Michael Rostás, Daniel Ruf, Vanessa Zabka, Ulrich Hildebrandt

University of Würzburg, Department of Botany II,

Julius-von-Sachs-Platz-3, D-97082 Würzburg, Germany.

Postprint version.

Original publication: Naturwissenschaften (2008), 10:992-1004

Corresponding author:

M. Rostás

e-mail: michael.rostas@botanik.uni-wuerzburg.de

Tel.: +49-931-888 6223

Fax: +49-931-888 6235 
Abstract The plant surface is the substrate upon which herbivorous insects and natural enemies meet and thus represents the stage for interactions between the three trophic levels. Plant surfaces are covered by an epicuticular wax layer which is highly variable depending on species, cultivar or plant part. Differences in wax chemistry may modulate ecological interactions. We explored whether caterpillars of Spodoptera frugiperda, when walking over a plant surface, leave a chemical trail (kairomones) that can be detected by the parasitoid Cotesia marginiventris. Chemistry and micromorphology of cuticular waxes of two barley eceriferum wax mutants (cer-za.126, cer-yp.949) and wild type cv. Bonus (wt) were assessed. The plants were then used to investigate potential surface effects on the detectability of caterpillar kairomones. Here we provide evidence that $C$. marginiventris responds to chemical footprints of its host. Parasitoids were able to detect the kairomone on wild type plants and on both cer mutants but the response to cer-yp.949 (reduced wax, high aldehyde fraction) was less pronounced. Experiments with caterpillar-treated wt and mutant leaves offered simultaneously, confirmed this observation: no difference in wasp response was found when wt was tested against cer-za.126 (reduced wax, wt-like chemical composition) but wt was significantly more attractive than cer-yp.949. This demonstrates for the first time that the wax layer can modulate the detectability of host kairomones.

Keywords Cotesia marginiventris $\cdot$ Epicuticular wax $\cdot$ Footprints $\cdot$ Host finding $\cdot$ Kairomone 


\section{Introduction}

Parasitoid insects are faced with the task of finding a suitable host that can be exploited as a food source by their offspring. This process of host foraging usually consists of several phases known as host habitat location, host location, host recognition, and host acceptance (Steidle and Van Loon 2002; Vinson 1998). At a distance, parasitoids of herbivorous insects use visual signals and volatile chemicals emitted by the infested plant as long-range cues (Turlings and Wäckers 2004; Vet and Dicke 1992). Once landed on a plant, multiple non- or low volatile searching stimulants that are linked to the host are used for retrieval. In response to these signals, female parasitoids often display characteristic sequences of walking that alternate with intense antennation and orthokinetic (changes in speed) and klinokinetik (changes in frequency of turning) responses - a behaviour that results in patchrestricted search. Potential sources of host location cues are e.g. host silk, faeces, pheromones or defence secretions (Steidle and Van Loon 2002). But, parasitoids are also known to eavesdrop on chemical footprints left by herbivores while walking over the substrate (e.g. Colazza et al. 1999; Conti et al. 2003; Klomp 1981). The chemistry of the footprints and in particular the identities of the kairomones remain to be elucidated. At least in the bug Nezara viridula cuticular hydrocarbons from the body, that are also present in the tarsi, seem to play a role as infochemicals for the egg parasitoid Trissolcus basalis (Colazza et al. 2007).

So far, experiments demonstrating the exploitation of host footprints by parasitoids have been performed using artificial substrate such as filter paper (but see Klomp 1981). However, in nature the interface where tritrophic interactions take place is often the surface or more specifically, the cuticle of a plant (Müller and Riederer 2005). The cuticle proper contains two different wax portions: the intracuticular waxes embedded in the cutin matrix and a layer of epicuticular waxes encrusted above. In some plant species epicuticular waxes form characteristic crystals that are mainly composed of the single predominant wax component (Barthlott et al. 1998). Chemical composition and amount of plant cuticular waxes may vary greatly depending on species, genotype or even within 
plant parts. In turn, this variation can modulate the outcome of many interactions between plants, herbivores and their natural enemies, for instance by providing feeding stimulants or deterrents and affecting insect attachment (Eigenbrode and Espelie 1995; Jetter et al. 2006). Furthermore, the hydrophobic plant surface can adsorb or absorb insect-derived organic compounds that may serve as delayed signals to conspecifics or other species (Noldus et al. 1991; Rostás and Hilker 2002; Eltz 2006; Wilms and Eltz 2008). The role of the plant surface chemistry in retaining host kairomones and thus in influencing the foraging behaviour of parasitoids has not yet been explored.

In this study we first assessed whether caterpillars of Spodoptera frugiperda (Lepidoptera: Noctuidae) leave chemical footprints on the epicuticular wax layer of a host plant that can be subsequently detected by Cotesia marginiventris (Hymenoptera: Braconidae), a specialist larval parasitoid of noctuid moths. Having established a role for such a kairomone in host location, we then used two eceriferum (cer) barley wax mutants and their corresponding wild type to investigate the effect of different plant surfaces on the kairomone perception of the parasitoid.

\section{Material and Methods}

Insects

Experiments were carried out with secondinstar caterpillars of Spodoptera frugiperda and mated 3-6-day old females of Cotesia marginiventris with no oviposition experience to test the wasp's innate response. Insects were reared as described in Winter and Rostás (2008)

Plants and wax analyses

Two barley cer mutants (cer-za.126, ceryp.949) known to be affected in the wax coating of their leaves (obtained from Nordic Gene Bank, Alnarp and Udda Lundqvist, Svalöv, both Sweden) and cv. Bonus wild type (wt) plants were analysed with respect to surface micromorphology, wax chemistry and wax amount. Waxes were extracted by dipping entire leaves into chloroform and were subsequently derivatized with bis-N,Otrimethylsilyltrifluoroacetamide (MachereyNagel, Düren, Germany). Individual wax components were identified and quantitated by gas chromatography (GC-MS and GC-FID). 
Wax crystal structure was assessed by scanning electron microscopy (SEM) and surface hydrophobicity was determined by contact angle measurements. Five replicates were carried out for each plant type and all analyses. For details see Zabka et al. (2008).

Behavioural assays

Behavioural choice experiments were performed to test whether C. marginiventris can detect chemical footprints of its host and to assess if variations in the epicuticular wax layer of the plant surface would affect the parasitoid's response.

A $5.5 \mathrm{~cm}$ diameter glass Petri dish was used as test arena. The Petri dish was surrounded by a $25 \mathrm{~cm}$ high white cardboard cylinder and illuminated by a 40W light bulb from above. The antennation behaviour of single wasps was observed for 10 minutes using the software Noldus Observer 5.0 (Noldus Information Technologies Inc., Wageningen, Netherlands). Only insects spending more than $50 \%$ of the time in motion were used for analyses. Wasps showing no antennation behaviour throughout the experiment were subjected to a motivation test in which they were provided three second instar host larvae for oviposition. Only ovipositing wasps were used for subsequent analyses. Antennation on the cutting edges of the leaves was not recorded as C. marginiventris is known to respond to the green leaf volatiles released by damaged plant tissue (Hoballah and Turlings 2005). Fifteen wasps were observed in each experiment if not stated otherwise. All behavioural assays were analyzed using the Wilcoxon matched pairs test.

Experiment 1: Detection of caterpillar footprints on different leaf surfaces

Two clip cages (diam.: $20 \mathrm{~mm}, \mathrm{~h}: 26 \mathrm{~mm}$ ), covered with gauze on top and bottom ends for ventilation, were attached side by side to the upper part of the second leaf of a potted barley plant (wt, cer-za.126 or cer-yp.949). One clip cage contained four small caterpillars $\left(\mathrm{L}_{2}\right)$ the second cage remained empty. Caterpillars were left to walk over the leaf surface for $15 \mathrm{~min}$. Clip cages were then removed and the leaf was visually examined. Only leaves without bite marks, faeces or silk were used in the experiment. Leaves that showed contaminations were discarded. The upper part 
of the leaf $(5 \mathrm{~cm}$ length), covering the caterpillar-treated and control areas, was cut off and placed into the arena for observation of parasitoid antennation behaviour.

In a second series of experiments a caterpillartreated leaf from wt was offered simultaneously with a caterpillar-treated leaf of cer-za.126 and cer-yp.949, respectively. In all experiments leaf material and wasps were exchanged after each observation.

Experiment 2: Chemical signal or wax crystal abrasion?

An experiment was performed to assess whether parasitoids respond to a host-derived chemical cue rather than to mechanical footprints possibly caused by breaking off wax crystals while walking over the leaf surface. Four caterpillars were caged on a microscope glass slide with a silanized hydrophobic surface for $15 \mathrm{~min}$. An empty cage was clipped on the other half of the slide. After removal of the cages wasp antennation behaviour was observed as described above $(n=12)$.

\section{Results}

Surface properties of wild type and wax mutants

In barley plants, the chemical composition of leaf waxes does not differ between adaxial and abaxial side or between epi- and intracuticular wax portions (Zabka et al. 2008). The cuticular wax of wild type barley plants (cv. Bonus) consisted of $75.8 \pm 1.4 \%$ long chain primary alkanols (Fig. 1), which in turn were dominated by the single compound hexacosanol $\left(\mathrm{C}_{26}\right)$. Further wax fractions consisted of esters $(11.5 \pm 1.4 \%)$, acids $(0.4 \pm$ $0.2 \%)$, aldehydes $(6.2 \pm 0.7 \%)$, n-alkanes $(1.0$ $\pm 0.1 \%$ ), and a fraction of unidentified compounds $(5.3 \pm 0.7 \%)$. Both cer mutants were characterized by considerably reduced total wax amounts and decreased alkanol fractions $($ cer-za.126 $=54.2 \pm 2.0 \%$, ceryp.949: $45.1 \pm 4.5 \%$ of total wax). Apart from the reduced main fraction, the composition of cer-za.126 was rather similar to wt (Fig. 2). In contrast, analyses of cer-yp.949 revealed a remarkably large fraction of aldehydes which mainly consisted of hexacosanal (cer-yp.949= 


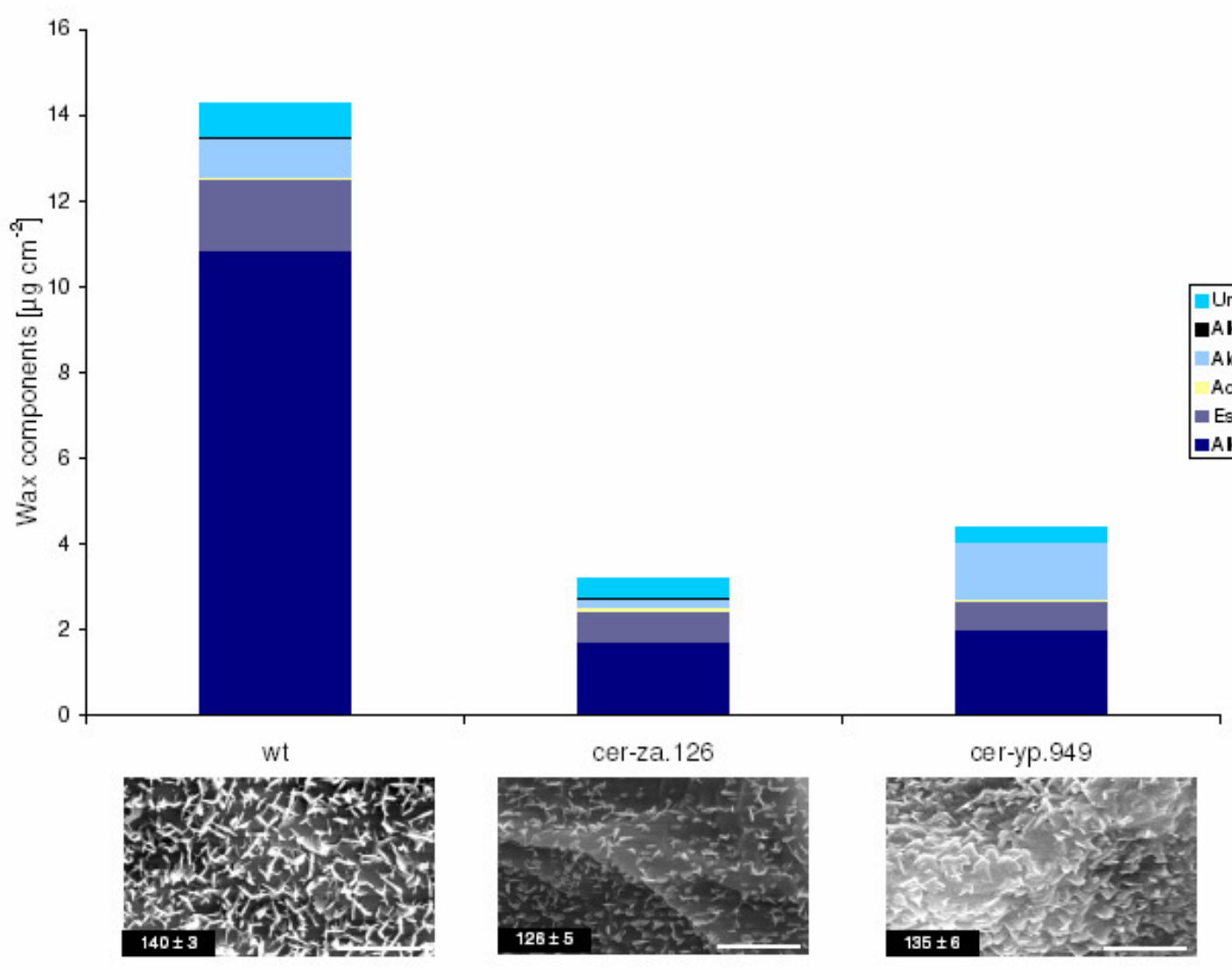

Figure 1 Cuticular wax compound class composition of barley (Hordeum vulgare cv. Bonus) secondary leaves from wild type (wt) and two eceriferum (cer) mutants. Bars represent mean values $(n=5)$. Below: scanning electron microscope pictures (SEM) of barley adaxial leaf surfaces. Wax crystal structures are depicted. Contact angles are given as means \pm SD. Bars, $2 \mu \mathrm{m}$.

$29.8 \pm 1.0 \%$, cer-za. $126=6.9 \pm 1.3 \%$, wt $=6.2$ $\pm 0.7 \%$ of total wax).

Micromorphology of epicuticular wax crystals was unique for each of the three barley lines (Fig. 1, SEM pictures). Abundant crystal platelets, vertically protruding $1-1.5 \mu \mathrm{m}$ from the leaf surface were characteristic for wt plants. The surface of cer-za.126 was rather smooth as it exhibited a reduced number of small-sized platelets. The leaf surface of ceryp.949 was densely covered by plate-like crystals which were more horizontally oriented, thus giving it a rough appearance.

Surface hydrophobicity was similarly high in wt $\left(140^{\circ} \pm 3\right)$ and cer-yp.949 $\left(135^{\circ} \pm 3\right)$ and moderatly lower in cer-za.126 $\left(126^{\circ} \pm 5\right)$. 


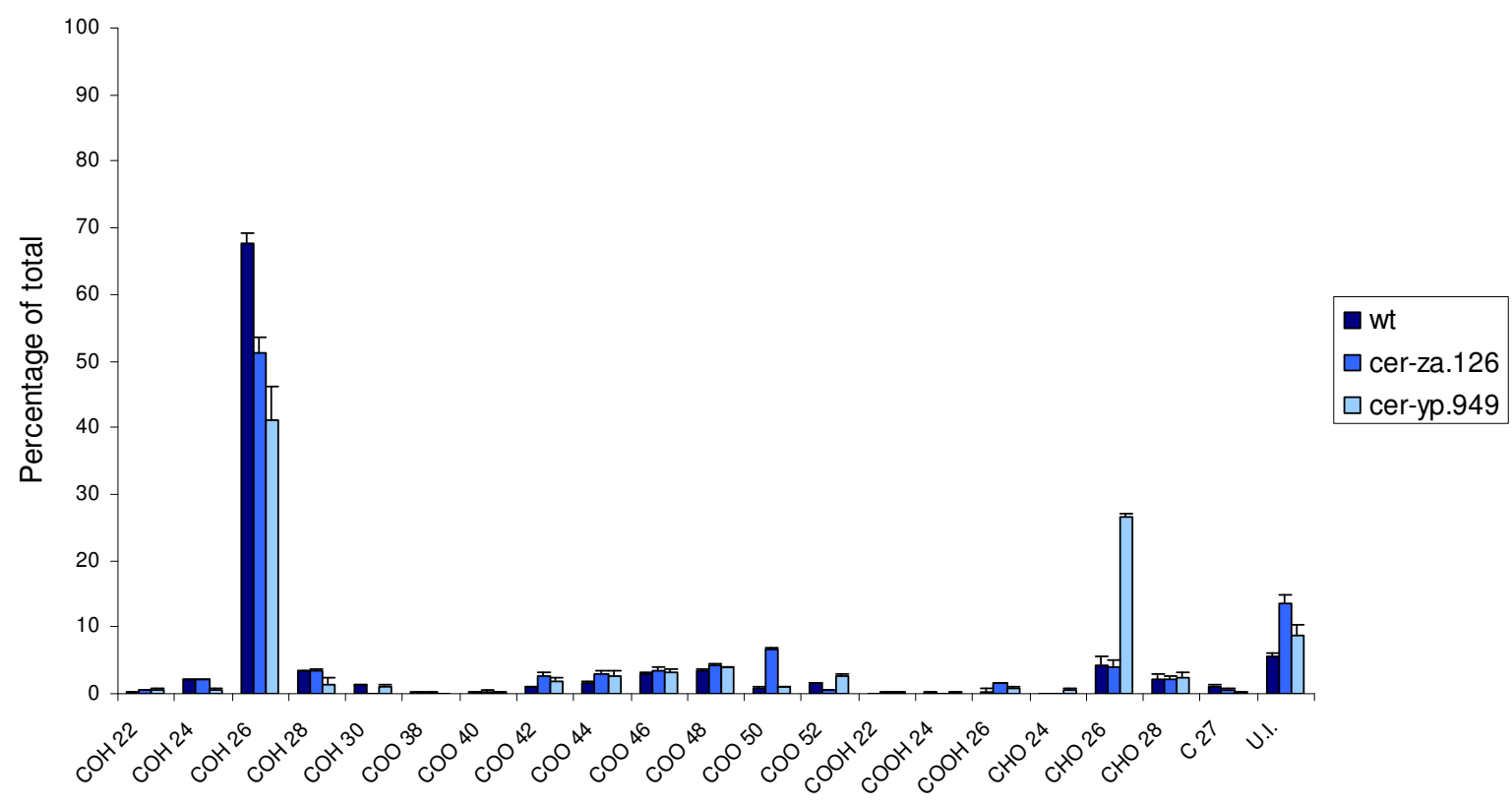

Figure 2 Relative amounts of barley leaf wax compounds. Wt = wild type (cv Bonus), cer-za.126 and cer-yp.949= eceriferum wax mutants. $\mathrm{COH}=$ primary alkanol, $\mathrm{COO}=$ ester, $\mathrm{COOH}=$ fatty acid, $\mathrm{CHO}=$ aldehyde, $\mathrm{C}=$ alkane, U.I. $=$ unidentified. Numbers behind letters indicate chain length. Bars and whiskers represent means and standard deviations.

Experiment 1: Detection of caterpillar footprints on different leaf surfaces

Female parasitoids displayed prolonged characteristic antennation behaviour on all leaf areas where caterpillars of S. frugiperda had been walking on previously (Fig. 3a). In wt and cer-za.126 the preference for the treated area was significant compared to the control side (Wilcoxon matched pairs test, wt: $\mathrm{Z}=$ 3.64, $\mathrm{P}<0.001, \mathrm{n}=15$; cer-za.126: $\mathrm{Z}=2.70, \mathrm{P}$ $<0.001, \mathrm{n}=15)$. Wasps also preferred the treated area on cer-yp.949 but difference in antennation time was less pronounced (Wilcoxon matched pairs test, $\mathrm{Z}=2.27, \mathrm{P}=$ $0.02, \mathrm{n}=15)$.

When caterpillar-treated wt leaves were tested against both wax mutants, parasitoids did not antennate significantly longer on wt than on cer-za.126 (Wilcoxon matched pairs test, $\mathrm{Z}=$ 1.54, $\mathrm{P}=0.12, \mathrm{n}=15$, Fig. 3b). However, $\mathrm{a}$ significant preference for wt was found when compared to cer-yp.949 (Wilcoxon matched pairs test, $\mathrm{Z}=2.44, \mathrm{P}=0.015, \mathrm{n}=15$ ). 


\section{a}

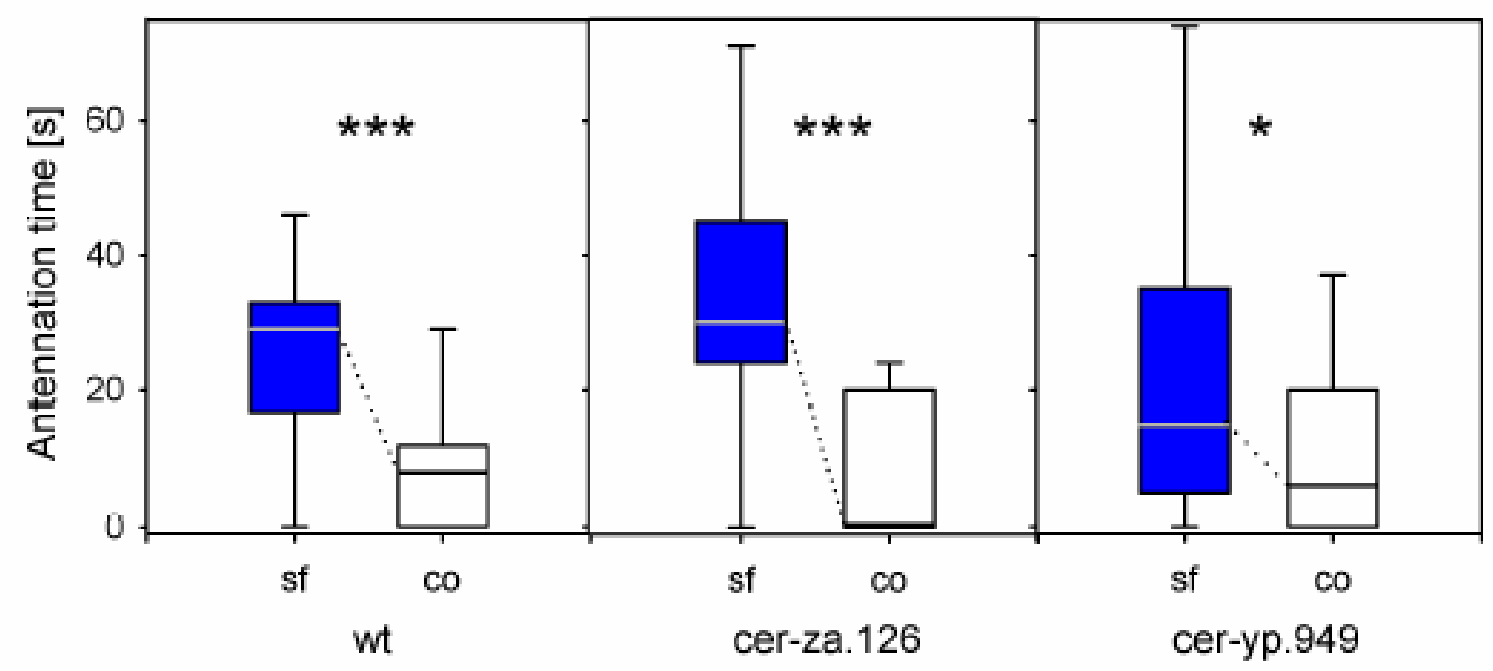

b

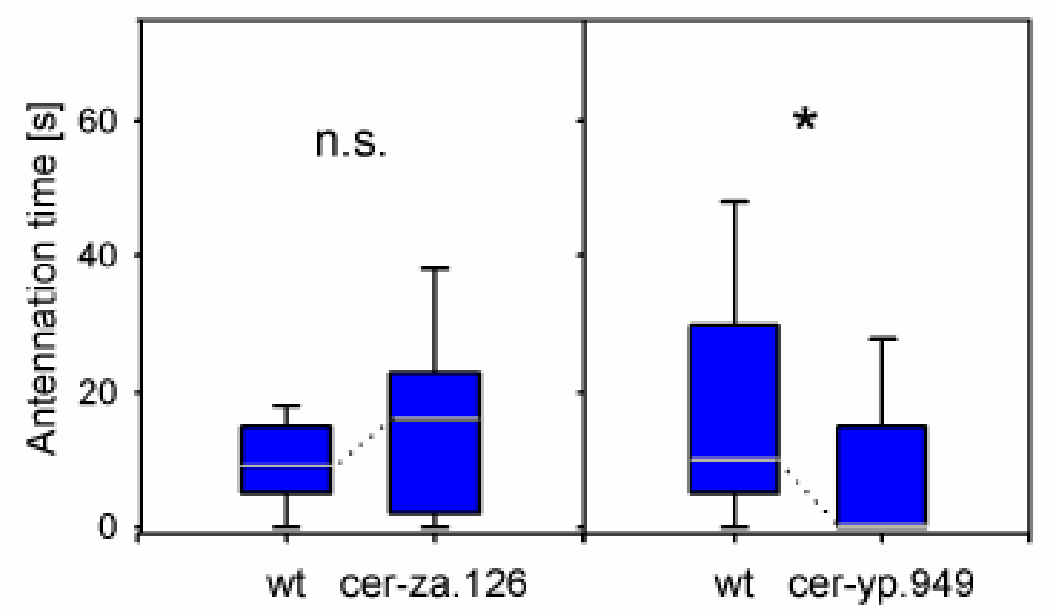

Figure 3 Antennation behaviour of female Cotesia marginiventris on leaf section from barley (Hordeum vulgare cv. Bonus). Leaf sections were taken from wild type (wt) and two eceriferum (cer-za.126, cer-yp.949) mutants, respectively. a Four caterpillars $\left(\mathrm{L}_{2}\right)$ of Spodoptera frugiperda had walked upon one half of the leaf for $15 \mathrm{~min}$ (sf), the other half remained untreated as a control (co). b Caterpillar-treated wild type (wt) leaves were tested against caterpillar-treated mutant leaves. Boxes show median (line), $25^{\text {th }}$ and $75^{\text {th }}$ percentiles. Whiskers are $5^{\text {th }}$ and $95^{\text {th }}$ percentiles. Asterisks denote significant differences: $\quad * * * P<0.001, \quad<P<0.05, \quad$ n.s. $\quad<\quad$ not significant 
Experiment 2: Chemical signal or wax crystal abrasion?

Female C. marginiventris antennated exclusively on the caterpillar-treated side of the silanized glass slide (Wilcoxon matched pairs test, caterpillar-treated area; median: 12.0 $\mathrm{s}, 25^{\text {th }}$ percentile: $7.3 \mathrm{~s}, 75^{\text {th }}$ percentile: $21.5 \mathrm{~s}$, $\mathrm{Z}=3.06, \mathrm{P}=0.002, \mathrm{n}=12$ ).

\section{Discussion}

The host finding behaviour of Cotesia marginiventris, a parasitoid of noctuid larvae, is well defined and a considerable body of literature exists on the chemical cues used for host habitat (plant volatiles; e.g. Turlings et al. 1990) and host location (faeces, exuviae, moth scales; e.g. Loke and Ashley 1984). We extend this knowledge by showing that female C. marginiventris can perceive chemical footprints left by caterpillars of Spodoptera frugiperda when walking over a leaf surface.

The only parts we observed to be in contact with the substrate were the prolegs, the claspers and the claws of the thoracical legs of the herbivore. Therefore, we propose that the most likely source of the residue substances should be the prolegs and claspers as they are covered by a cuticle like the rest of the body. Due to the lipophilic properties of the plant cuticle only lipophilic uncharged organic chemicals can be sorbed onto a plant surface and stay there for some time (Müller and Riederer 2005). Hexane washings from microscopic slides that were walked over by caterpillars of S. frugiperda elicited antennation behaviour and contained the same long chain hydrocarbons (mainly C21-32 alkanes) as hexane extracts from the caterpillar cuticle (Rostás, unpublished data). Cuticular hydrocarbons are also known from other herbivore-parasitoid associations to serve as kairomones (Borges et al. 2003; Peri et al. 2006). Substances derived from the claws of the true legs cannot be excluded as kairomones but the claws touch only a very small area of the leaf. The fact that $\mathrm{C}$. marginiventris uses chemicals and not merely physical cues that may have resulted for instance from the abrasion of wax crystals is evident since wasps displayed clear antennation behaviour on the caterpillar-treated area of a hydrophobic glass surface. 
To answer the question whether the physicochemical properties of the epicuticular wax layer may affect the detectability of host kairomones by C. marginiventris, it was necessary to characterize the leaf surface of a variety of cer mutants with reduced wax accumulation (Zabka et al. 2008). The two lines we used for behavioural assays were selected because one mutant (cer-za.126) had a rather similar wax composition compared to wt cv. Bonus but had a smooth surface while the other (cer-yp.949) showed a markedly increased aldehyde fraction and presented a rough surface that was densely covered by horizontally-oriented wax platelets (Fig. 1 and 2).

Parasitoids were able to recognize the former presence of their host on the three different leaf surfaces. However, in contrast to the strong antennation behaviour observed on the caterpillar-treated area of wt and cer-za.126 leaves, the parasitoid's preference for host substances was less pronounced on leaves of cer-yp.949 (Fig. 3a). We further confirmed this notion in experiments where parasitoids were able to directly compare caterpillar-treated leaf sections from wt and mutant barley (Fig. 3b). The significant preference for wt over cer- yp.949 is related to chemical and/or physical differences in the wax surface but may result from a number of mechanisms. Host compounds could be adsorbed or absorbed to the plant cuticles to varying extents, i.e. the surface of cer-yp.949 may have a weaker affinity for the relevant bioactive substances. Furthermore, a unique trait of cer-yp.949 is the large proportion of long chain aldehydes in the wax layer. This modified chemical composition of the leaf surface could have affected the wasp's perception of the kairomone. It is also conceivable that the chemical composition affected the retention of caterpillar-derived compounds. Differential detection of the kairomone due to an overlap in the same compounds from plant wax and insect footprints cannot be ruled out. However, the only compound in question, heptacosane $\left(\mathrm{C}_{27}\right)$, is a very minor wax component and does not vary much between wt and mutants (see Fig. 2). Wax crystals are abundant on wt and cer-yp.949 surfaces but scarce on cer-za.126. However, in wt and cer-yp.949 they are different in orientation and may thus have somehow affected parasitoid foraging. On the other hand, wt and cer-yp.949 leaves show very similar degrees of surface hydrophobicity 
(expressed as contact angle), which means that this trait does not explain the effect. Since parasitoids did not distinguish between wt and cer-za.126, total amount of wax was not decisive for kairomone recognition. Further experiments will have to decipher whether differential detection of the kairomone was due to the chemical properties of the waxes alone and/or the physical structures of the crystals, whose shape depend on the wax composition. The epicuticular wax layer of plants has been shown to influence the foraging success of natural enemies (Eigenbrode 2004). For example, on wax-reduced pea mutants, Aphidius ervi spent more time actively foraging and a larger number of aphids were parasitized than on wt plants (Chang et al. 2004). The main factor with respect to plant waxes that is thought to determine the foraging success of predators or parasitoids is their ability to attach to the plant surface. Reduced wax blooms often correlate with better insect attachment to the plant surface (Eigenbrode and Jetter 2002). Concluding from our data we suggest that in addition to attachment differences, potential effects of the wax layer on kairomone detectability should also be taken into consideration when evaluating the foraging success of natural enemies. Using crop plant varieties with appropriate wax surfaces may enhance the efficiency of parasitoids and could thus improve the biological control of pests.

\section{Acknowledgements}

We thank Markus Riederer for providing lab space and Jutta Winkler-Steinbeck for cultivating barley plants. Funding from the Deutsche Forschungsgemeinschaft (DFG, SFB 567, TP B9 and TP A5) is acknowledged. 


\section{References}

Barthlott W, Neinhuis C, Cutler D, Ditsch F, Meusel I, Theisen I, Wilhelmi, H (1998) Classification and terminology of plant epicuticular waxes. Bot J Linn Soc 126:237-260

Borges M, Colazza S, Ramirez-Lucas P, Chauhan KR, Moraes MCB, Aldrich JR (2003) Kairomonal effect of walking traces from Euschistus heros (Heteroptera: Pentatomidae) on two strains of Telenomus podisi (Hymenoptera: Scelionidae). Physiol Entomol 28:349-355

Chang GC, Neufeld J, Durr D, Duetting PS, Eigenbrode SD (2004) Waxy bloom in peas influences the performance and behavior of Aphidius ervi, a parasitoid of the pea aphid. Entomol Exp Appl 110:257-265

Colazza S, Aquila G, De Pasquale C, Peri E, Millar JG (2007) The egg parasitoid Trissolcus basalis uses n-nonadecane, a cuticular hydrocarbon from its stink bug host Nezara viridula, to discriminate between female and male hosts. J Chem Ecol 33:1405-1420

Colazza S, Salerno G, Wajnberg E (1999) Volatile and contact chemicals released by Nezara viridula (Heteroptera: Pentatomidae) have a kairomonal effect on the egg parasitoid Trissolcus basalis (Hymenoptera: Scelionidae). Biol Control 16:310-317

Conti E, Salerno G, Bin F, Williams HJ, Vinson SB (2003) Chemical cues from Murgantia histrionica eliciting host location and recognition in the egg parasitoid Trissolcus brochymenae. J Chem Ecol 29:115-130

Eigenbrode SD (2004) The effects of plant epicuticular waxy blooms on attachment and effectiveness of predatory insects. Arthropod Struct Dev 33:91-102

Eigenbrode SD, Espelie KE (1995) Effects of plant epicuticular lipids on insect herbivores. Annu Rev Entomol 40:171-194

Eigenbrode SD, Jetter R (2002) Attachment to plant surface waxes by an insect predator. Integr Comp Biol 42:1091-1099

Eltz T (2006) Tracing pollinator footprints on natural flowers. J Chem Ecol 32:907-915

Hoballah ME, Turlings TCJ (2005) The role of fresh versus old leaf damage in the attraction of parasitic wasps to herbivore-induced maize volatiles. J Chem Ecol 31:2003-2018

Jetter R, Kunst L, Samuels AL (2006) Composition of plant cuticular waxes. In: Riederer M, Müller C (eds) Biology of the plant cuticle. Blackwell Publishing, Oxford

Klomp H (1981) Parasitic wasps as sleuthhounds - Response of an ichneumon wasp to the trail of its host. Netherlands J Zool 31:762-772 
Loke WH, Ashley TR (1984) Sources of fall armyworm, Spodoptera frugiperda (Lepidoptera, Noctuidae), kairomones eliciting host-finding behavior in Cotesia (=Apanteles) marginiventris (Hymenoptera, Braconidae). J Chem Ecol 10:1019-1027

Müller C, Riederer M (2005) Plant surface properties in chemical ecology. J Chem Ecol $31: 2621-2651$

Noldus LPJJ, Potting RPJ, Barendregt HE (1991) Moth sex pheromone adsorption to leaf surface: bridge in time for chemical spies. Physiol Entomol 16:329-344

Peri E, Sole MA, Wajnberg E, Colazza S (2006) Effect of host kairomones and oviposition experience on the arrestment behavior of an egg parasitoid. J Exp Biol 209:3629-3635

Rostás M, Hilker M (2002) Feeding damage by larvae of the mustard leaf beetle deters conspecific females from oviposition and feeding. Entomol Exp Appl 103:267-277

Steidle JLM, Van Loon JJA (2002) Chemoecology of parasitoid and predator oviposition behaviour. In: Hilker M, Meiners T (eds) Chemoecology of insect eggs and egg deposition. Blackwell Publishing, Berlin, pp 291-317

Turlings TCJ, Tumlinson JH, Lewis WJ (1990) Exploitation of herbivore-induced plant odors by host-seeking parasitic wasps. Science 250:1251-1253

Turlings TCJ, Wäckers F (2004) Recruitment of predators and parasitoids by herbivore-injured plants. In: Cardé RT, Millar J (eds) Advances in insect chemical ecology. Cambridge University Press, Cambridge

Vet LEM, Dicke M (1992) Ecology of infochemical use by natural enemies in a tritrophic context. Annu Rev Entomol 37:141-172

Vinson SB (1998) The general host selection behavior of parasitoid hymenoptera and a comparison of initial strategies utilized by larvaphagous and oophagous species. Biol Control 11:79-96

Wilms J, Eltz T (2008) Foraging scent marks of bumblebees: footprint cues rather than pheromone signals. Naturwissenschaften 95:149-153

Winter TR, Rostás M (2008) Ambient ultraviolet radiation induces protective responses in soybean but does not interfere with tritrophic interactions. Environ Pollut 155:290-297

Zabka V, Stangl M, Bringmann G, Vogg G, Riederer M, Hildebrandt U (2008) Host surface properties affect prepenetration processes in the barley powdery mildew fungus. New Phytol $177: 251-263$ 